\title{
Surface Modifications: Evaporation of Oxide Islands on Metal Surfaces Investigated by In situ TEM
}

\section{Guangwen Zhou, Judith C. Yang}

Department of Materials Science and Engineering, University of Pittsburgh,Pittsburgh, PA 15260

Controlled nanoscale pattern formation on surfaces has become one of the most important and challenging areas of nanoscience. Recently, we have demonstrated the potential for a new method for the production of nanostructured oxide island arrays by controlled oxidation of solid surfaces $[1,2,3]$. The opportunity exists to provide important advances in a variety of technologies via exploitation of the potentially novel optical, magnetic and sensor properties of these metal-oxide arrays [4]. In this work, we present a new method for surface modifications: evaporation of oxide islands on metal surfaces and we show how detailed the in situ TEM measurements of surface morphology made during the oxide evaporation can be used to determine the kinetics of the evaporation process.

Single crystal $99.999 \%$ pure $700 \AA \mathrm{Cu}(100)$ films were oxidized in a modified UHV JEOL200 CX. Dramatically different morphologies of oxide nanostructures can be achieved by an appropriate choice of the oxidation temperature [2, 3]. The evolution of $\mathrm{Cu}_{2} \mathrm{O}$ islands morphology under isothermal annealing was followed inside the microscope, and significant morphological changes were observed. Fig. 1 shows the evaporation of the pyramids at the $1000^{\circ} \mathrm{C}$, where the original oxide islands were formed at $800^{\circ} \mathrm{C}$. The original islands had a square shape and the normal to the edges of the islands are along $<110>$ directions. The evaporation started from the island corners as shown in Fig. 1a, and left multi-facet trenches at the corners. With the continuous evaporation of the island, $<100>$ edges formed and increased its size gradually. The size of the $<110>$ edges became smaller and transformed to round corners. The bright contrast along the perimeter of the island can be interpreted as thinned regions due to the evaporation of $\mathrm{Cu}_{2} \mathrm{O}$ islands. We can find that the thinned regions became larger with the continuous evaporation of the island. The trench morphology along the perimeter of the island also evolved gradually from multiple facets to V-shape. Fig. 2(a) shows the surface morphology after the complete evaporation of the islands, and Fig. 2(b) is an AFM image which clearly shows the notch structures on the surface due to the oxide evaporation. This observation demonstrates the evaporation of oxide islands is an effective way to create notch arrays on metal surfaces which allows one to modify the surface topology and has potential applications in nanotechnology.

Evaporation is an intrinsically nonequilibrium process that can be understood only when considered as a function of both space and time. We can calculate that the rate of change of the radius $r$ is related to the evaporation time $t$ based on Kelvin effect [5]

$$
\frac{d r}{d t}=0.97 \times 10^{5} \sqrt{\frac{M}{T}} \exp \left(-\frac{\Delta H_{e}}{R T}+I\right) \exp \left(\frac{2 \sigma M}{\rho R T r}\right)
$$


Applying this equation to the experimental data from the in situ observation, the surface energy of the $\mathrm{Cu}_{2} \mathrm{O}$ islands could be determined. This experimental observation also gives a direct evidence of the Kelvin effect. Usually the surface energies of oxides are difficult to obtain, therefore, this work also allows one to estimate experimentally the surface energies of the oxide islands.

This research is funded by the NSF (DMR \# 9902863) and DOE (DEFG02-96ER45439). The experiments were performed at the Materials Research Laboratory, UIUC. The authors thank Ray Twesten, Kevin Colrary for their help.

\section{Reference:}

[1] G.W. Zhou, J. C. Yang, Phys. Rev. Lett. 89, 6101(2002)

[2] G.W. Zhou, J.C. Yang, Appl. Surf. Sci. in press (2003)

[3] G.W. Zhou, J. C. Yang, Proc. Mater. Res. Soc., in press (2003)

[4] Aggarwal, S., Monga, A.P., Perusse, et al., Science 287, 2235 (2000)

[5] Blackman, M., Lisgarten, N.D., Skimmer, L.M., Nature 217, 1245 (1968)
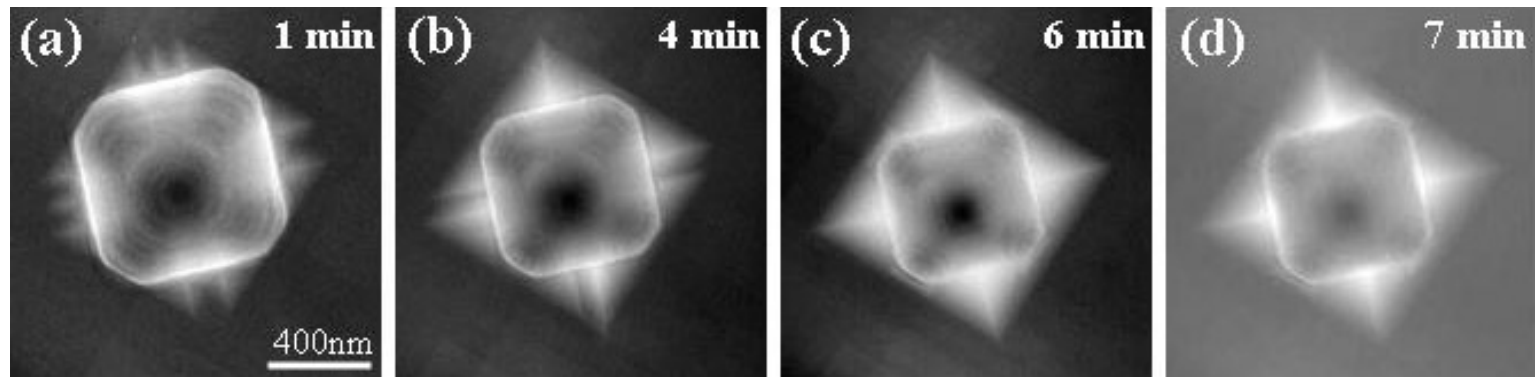

Figure 1. The in situ evaporation of an island at $1000^{\circ} \mathrm{C}$, where the original oxide islands are formed at $800^{\circ} \mathrm{C}$.
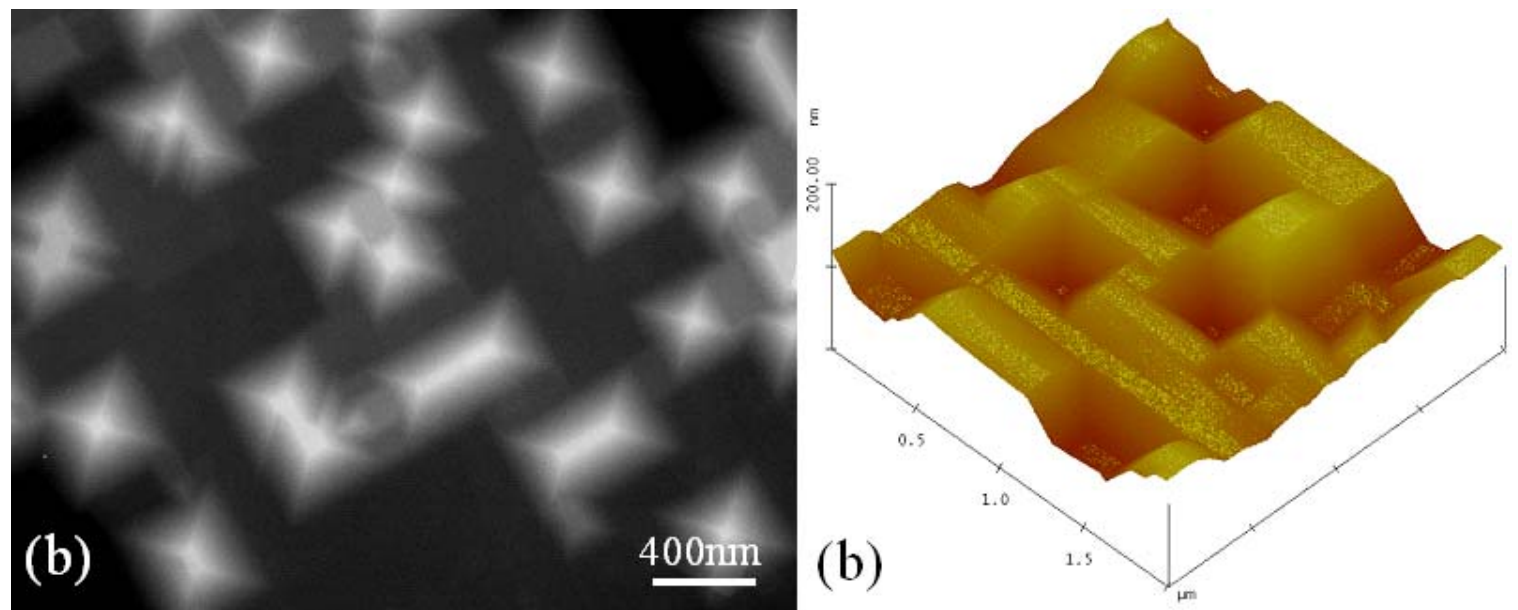

Figure 2. (a) The morphology of $\mathrm{Cu}(100)$ film after the complete evaporation of the $\mathrm{Cu}_{2} \mathrm{O}$ islands at $1000^{\circ} \mathrm{C}$; (b) $\mathrm{AFM}$ image of the surface topography of the $\mathrm{Cu}$ film with the complete evaporation of the oxide islands. 